\title{
Correction to: A Range of Earth Observation Techniques for Assessing Plant Diversity
}

\author{
Angela Lausch, Marco Heurich, Paul Magdon, Duccio Rocchini, \\ Karsten Schulz, Jan Bumberger, and Doug J. King
}

\section{Correction to:}

\section{Chapter 13 in: J. Cavender-Bares et al. (eds.),} Remote Sensing of Plant Biodiversity, https://doi.org/10.1007/978-3-030-33157-3_13

The original version of this book was inadvertently published with an incorrect affiliation "Humboldt University to Berlin, Geography Department, Leipzig, Germany, Humboldt Universität zu Berlin, Geography Department, Berlin, Germany, e-mail: angela.lausch@ufz.de” of the 13th chapter author Angela Lausch.

The correct affiliation "Department Computational Landscape Ecology, Helmholtz Centre for Environmental Research-UFZ, Leipzig, Germany, Geography Department, Humboldt University Berlin, Berlin, Germany, e-mail: angela.lausch@ ufz.de" has been updated in 13th chapter.

The updated online version of this chapter can be found at https://doi.org/10.1007/978-3-030-33157-3_13 